\title{
EchoGéo
}

$57 \mid 2021$

Les enjeux de l'alimentation en eau potable des villes

\section{Sourciers des villes}

Editorial

Serge Weber

\section{(2) OpenEdition}

Journals

Édition électronique

URL : https://journals.openedition.org/echogeo/22564

DOI : $10.4000 /$ echogeo.22564

ISSN : 1963-1197

Éditeur

Pôle de recherche pour l'organisation et la diffusion de l'information géographique (CNRS UMR 8586)

Référence électronique

Serge Weber, «Sourciers des villes », EchoGéo [En ligne], 57 | 2021, mis en ligne le 03 novembre 2021, consulté le 05 novembre 2021. URL : http://journals.openedition.org/echogeo/22564 ; DOI : https:// doi.org/10.4000/echogeo.22564

Ce document a été généré automatiquement le 5 novembre 2021.

EchoGéo est mis à disposition selon les termes de la licence Creative Commons Attribution - Pas d'Utilisation Commerciale - Pas de Modification 4.0 International (CC BY-NC-ND) 


\title{
Sourciers des villes
}

\author{
Editorial
}

\section{Serge Weber}

1 Pour son troisième numéro de l'année 2021, Echogéo propose de remonter à proprement parler aux sources des villes, qui sont confrontées aux multiples défis de leur approvisionnement en eau potable. Émilie Lavie et Mathilde Resch, coordinatrices du dossier thématique de la rubrique Sur le champ, ont pris leur baguette de coudrier pour nous guider dans l'exploration des espaces concernés par le cycle urbain de l'eau de diverses grandes villes, de Paris à La Paz, en passant par Bruxelles, Dijon, Ndjamena, Bogotà et Lima. Ce dossier confronte des situations de pays du Nord, où les systèmes d'approvisionnement des villes garantissent presque partout l'universalité de l'accès à la ressource mais doivent être entretenus et améliorés, à celles de pays du Sud où beaucoup de grandes villes n'ont d'autre choix que d'entériner après-coup des pratiques alternatives et plus ou moins informelles, formant des systèmes composites et parfois conflictuels.

2 C'est dans l'articulation entre ces deux grands types de situation que ce dossier apporte un regard renouvelé sur une question fondamentale pour toutes les villes. La précarité hydrique et la vulnérabilité hydrique existent bel et bien dans des métropoles de pays riches dont les réseaux techniques sont performants depuis longtemps, c'est ce que nous montrent Xavier May, Pauline Bacquaert, Jean-Michel Decroly, Léa de Guiran, Chloé Deligne, Pierre Lannoy et Valentina Marziali à propos de Bruxelles. Comme l'écrivait Catherine Carré en 2001, le cycle urbain de l'eau dans les pays du Nord n'est jamais complètement abouti car les problèmes que pose l'approvisionnement en eau des grandes villes sont de telle ampleur qu'ils réinterrogent sans cesse les échelles territoriales de sa gestion. En effet, les interactions avec les territoires, parfois lointains et disjoints, qui fournissent l'eau potable, malgré tous les cadres établis pour une bonne coopération, n'en sont pas moins des rapports de pouvoir qui viennent s'intercaler dans les maillages politiques et administratifs. L'exemple du renforcement, du fait de la gestion de l'eau, du pouvoir du niveau métropolitain à Dijon est à ce titre éloquent, comme on le voit dans l'analyse de Sandrine Petit, Marie-Hélène Vergote et Emmanuel Dumont. Explorant un bassin versant qui fournit une part non négligeable de l'eau 
potable des Parisiens, celui de la Vanne, dont la source se situe dans le département de l'Aube, Audrey Vincent et Philippe Fleury montrent que la municipalité de Paris joue, en vue d'améliorer la qualité de l'eau, un rôle de plus en plus décisif dans les choix d'orientation agricole et de méthodes culturales dans des espaces éloignés.

3 Le dossier éclaire également les limites de la transférabilité des modèles du Nord vers le Sud au travers des situations africaines ou sud américaines. Le "grand système ", modèle centralisé de gestion de l'eau potable à l'échelle d'une agglomération, n'apparaît pas toujours transposable. D'abord parce que les moyens à la fois techniques et financiers de la gestion métropolitaine ne suffisent pas pour se substituer à des solutions alternatives qu'on retrouve essentiellement dans les périphéries, à l'initiative des habitants. Ensuite parce que la coexistence de différents régimes d'adduction d'eau peut se révéler moins problématique qu'il n'y paraît, à la fois en termes de coût et d'interaction avec l'environnement. C'est ce que montrent deux articles: le premier d'Ismaël Maazaz, qui analyse la coexistence de régimes disparates à Ndjamena, où les «bricolages hydrauliques» manuels coexistent avec une gestion publique et des forages privés ; le deuxième d'Angela Osorio, qui montre à partir du cas de Bogotà que les systèmes alternatifs communautaires d'approvisionnement en eau potable dans les paramos environnant la capitale bolivienne ont un rôle indéniable dans la protection et la restauration du milieu naturel et donnent des arguments en faveur d'une approche intégrée de la nature, compatible avec l'échelle métropolitaine de gestion de l'eau. Les épisodes de tension climatique mettent à l'épreuve les villes de Lima et La Paz qui sont confrontées à des crises récurrentes dans l'approvisionnement en eau, comme nous le rappellent dans leur article Sébastien Hardy et Jérémy Robert. Non seulement les initiatives alternatives coexistent avec une surenchère de projets de captages lointains et coûteux, portés par des élites techniques et économiques qui forment des "hydrocraties locales ", mais elles permettent également d'en combler les lacunes.

Le tourisme est à l'honneur dans deux de nos rubriques, Sur l'écrit et Sur l'image, qui s'intéressent aux sources de la touristicité des lieux.

Dans leur interview pour la rubrique Sur l'écrit, Jean-Christophe Gay et Philippe Violier insistent, en évoquant l'ouvrage Tourisme en France, qu'ils ont coécrit avec deux autres spécialistes, Véronique Mondou et Philippe Duhamel, sur les étapes historiques de l'invention des lieux touristiques. Ils dévoilent le rôle crucial des touristes eux-mêmes qui, par leurs pratiques, ont contribué à transformer la géographie des espaces français. Partant du constat que le sens du tourisme, la «re-création», est la clef essentielle pour comprendre les trajectoires des lieux, ils proposent une régionalisation inédite, fondée sur la géohistoire des pratiques. C'est un des traits de l'originalité de leur démarche et de ce livre, qui fait le point sur le renouvellement épistémologique de la géographie du tourisme depuis le tournant amorcé par les premiers travaux de l'équipe MIT (Mobilités, itinéraires, tourismes).

6 Ce n'est plus une baguette de noisetier mais un bâton de pèlerin qu'il nous faut pour arpenter avec David Lagarde, Patricia Panegos et Sébastien Rayssac les chemins de Saint-Jacques dans la rubrique Sur l'image. Ils étayent la notion d'empreinte territoriale à partir d'un travail de recherche-action réalisé pour une exposition itinérante autour d'un "chemin devenu ressource", convoquant aussi bien cheminants qu'acteurs locaux. En analysant un riche matériau de discours à partir du cadastre, de cartographies narratives et de photographies, ils éclairent la façon dont l'engouement 
pour la pratique du cheminement a des effets en profondeur sur les espaces traversés et sur la fabrique des imaginaires géographiques.

7 La rubrique Sur le métier invite les géographes à explorer une méthodologie peu usitée, la textométrie. Le texte de Cécilia Comelli, Grégoire Le Campion et Marie JauffretRoustide présente quelques résultats d'un vaste programme de recherche sur la manière dont des logiciels d'exploration automatisée de corpus textuels de grande ampleur permettent de remonter aux sources de la fabrique de lieux communs ou de stéréotypes. Deux principaux outils, Iramuteq et Alceste, les amènent à préciser la manière dont les termes utilisés dans des articles de presse participent à associer usage de drogues, catégories de personnes et catégories de lieux. Ce texte vient nous rappeler que, de manière plus générale, les méthodes de traitement automatisé des langues, alors qu'elles sont encore peu utilisées dans le métier de géographe, réservent des possibilités multiples pour consolider une approche constructiviste de la spatialisation des faits sociaux. 